\title{
Polynomial Chaos Expansion and Steady-State Response of a Class of Random Dynamical Systems
}

\author{
E. Jacquelin ${ }^{1}$; S. Adhikari²; J.-J. Sinou ${ }^{3}$; and M. I. Friswell ${ }^{4}$
}

\begin{abstract}
The first two moments of the steady-state response of a dynamical random system are determined through a polynomial chaos expansion (PCE) and a Monte Carlo simulation that gives the reference solution. It is observed that the PCE may not be suitable to describe the steady-state response of a random system harmonically excited at a frequency close to a deterministic eigenfrequency: many peaks appear around the deterministic eigenfrequencies. It is proved that the PCE coefficients are the responses of a deterministic dynamical system - the so-called PC system. As a consequence, these coefficients are subjected to resonances associated to the eigenfrequencies of the PC system: the spurious resonances are located around the deterministic eigenfrequencies of the actual system. It is shown that the polynomial order required to obtain some good results may be very high, especially when the damping is low. These results are shown on a multidegree-of-freedom (DOF) system with a random stiffness matrix. A 1-DOF system is also studied, and new analytical expressions that make the PCE possible even for a high order are derived. The influence of the PC order is also highlighted. The results obtained in the paper improve the understanding and scope of applicability of PCE for some structural dynamical systems when harmonically excited around the deterministic eigenfrequencies. DOI: 10.1061/(ASCE)EM.1943-7889.0000856. ㅇ 2014 American Society of Civil Engineers.
\end{abstract}

Author keywords: Random systems; Structural dynamics; Polynomial chaos expansion; Steady-state response; Convergence.

\section{Introduction}

The consideration of uncertainties plays a crucial role in accessing the dynamic performance of a structure. A primary reason for this is the increasing parametric sensitivity of the dynamic response around the resonance frequencies. Response sensitivity to variations of the structure, such as parametric variation in the Young's modulus, Poisson's ratio, density, or other kind of error sources, e.g., errors in the model of damping, can be evaluated through uncertainty quantification. It is generally considered that, at low frequencies, the study of the response is best addressed by a parametric approach. For this case, the stochastic FEMs (SFEM) (Ghanem and Spanos 1991) can be applied to obtain statistics of response or the eigenvalues and eigenvectors of the system.

Stochastic FEMs are principally divided into simulation-based methods [e.g., Monte Carlo Simulation (MCS)] (Hurtado and Barbat 1998) and expansion-based methods (perturbation method, spectral approach, and stochastic reduced basis method). Reviews on SFEMs applied to calculate the response statistics are given, for example, in Stefanou (2009) and Schuëller and Pradlwarter (2009). Application

${ }^{1}$ Professor, Univ. de Lyon, F-69622 Lyon, France; Univ. Claude Bernard Lyon 1, F-69622 Villeurbanne, France; and Institut Français des Sciences et Technologies des Transports, de l'Aménagement et des Réseaux (IFSTTAR), Unité Mixte de Recherche (UMR)-T9406, Laboratoire de Biomécanique et Mécanique des Chocs, F-69675 Bron, France (corresponding author). E-mail: eric.jacquelin@univ-lyon1.fr

${ }^{2}$ Professor, College of Engineering, Swansea Univ., Swansea SA2 8PP, U.K.

${ }^{3}$ Professor, École Centrale de Lyon, Laboratoire de Tribologie et Dynamique des Systèmes, Unité Mixte de Recherche (UMR) Centre National de la Recherche Scientifique 5513, F-69134 Écully, France.

${ }^{4}$ Professor, College of Engineering, Swansea Univ., Swansea SA2 8PP, U.K.

Note. This manuscript was submitted on March 5, 2014; approved on August 1, 2014; published online on September 11, 2014. Discussion period open until February 11, 2015; separate discussions must be submitted for individual papers. This paper is part of the Journal of Engineering Mechanics, (C) ASCE, ISSN 0733-9399/04014145(11)/\$25.00. of perturbation methods to calculate response can be found in Kleiber and Hien (1992). Spectral approach methods are reviewed in Nouy (2009), where the most widely used spectral approach method is polynomial chaos (PC) using a Galerkin scheme (Ghanem and Spanos 1991) or collocation methods (Berveiller et al. 2006; Xiu and Hesthaven 2005; Xiu 2007). A different approach, followed in Udwadia (1987a, b), proposed exact analytical expressions for the response statistics for a single degree-of-freedom (1-DOF) system. They were obtained from the probability density function (PDF) of the eigenvalues, related to the PDF of the random parameters. Also, Laplace's integral has been used to calculate moments and reliability of response, where the maximum and Hessian of the logarithm of the integrated function are obtained through different numerical methods (Papadimitriou et al. 1997). In the context of random skeletal structures, a doubly spectral stochastic finite-element (Adhikari 2011) approach was developed in the frequency domain. This can be viewed as a stochastic dynamic stiffness formulation (Ostoja-Starzewski and Woods 2003; Manohar and Adhikari 1998; Adhikari and Manohar 2000).

The dynamic response of linear stochastic systems can be considered either in the modal domain or directly in the time or frequency domain. The review papers (Stefanou 2009; Pradlwarter and Schuëller 2010; Schuëller and Pradlwarter 2009) give an account of the various techniques available. In the modal domain, many authors used PC-based spectral projection approaches (Ghosh et al. 2005; Verhoosel et al. 2006; Pascual and Adhikari 2012) for the eigensolutions of the system. However, spectral methods have not been used widely for the direct computation of the response statistics. Pichler et al. (2009) proposed a mode-based metamodel for the frequency response functions of stochastic structural systems. Adhikari and Manohar (1999) proposed a random eigenfunction expansion method based on Galerkin projection for the frequency domain response of stochastic dynamical systems. Van den Nieuwenhof and Coyette (2003) proposed modal approaches for the stochastic dynamic response of structures with material and geometric uncertainties in the frequency domain. Impollonia and 
Ricciardi (2006) proposed a procedure to derive, in explicit form, the stationary response of a linear structure subjected to Gaussian white noise stochastic excitation. Their method is based on the solution of complex eigenvalue problems for each fluctuating parameter. Muscolino et al. (2000) discussed improved dynamic analysis of structures with parametric uncertainties under deterministic input forces. Fang (1995) proposed a transfer matrix-based approach in conjunction with a perturbation method for dynamical systems. Falsone and Ferro $(2005,2007)$ proposed the exact principal deformation mode (EPDM) approach for the frequency-domain response of a general linear dynamical system. Their results show excellent agreement with direct MCS results. Recently, Kundu and Adhikari (2014) proposed a spectral-function approach for the timedomain response of stochastic systems. The present work is not related to the time integration issues with PC expansion (PCE), as the excitation as well as the response are harmonic; so, the calculations are in the frequency domain and then, different issues arise. Lucor and Karniadakis also worked on nonlinear random systems (Lucor and Karniadakis 2004).

Few authors have discussed the convergence (Field and Grigoriu 2004, 2007) of the PCE for static problems (elliptic equations in general). Practical works on dynamic problems show that some of the nice and well-known features may not be preserved. There is a lack of a detailed investigation in the literature on the behavior of PCE in a dynamic context. The aim of this paper is to contribute so that PCE can be applied to uncertain dynamical systems in an accurate and computational-efficient manner. First, the authors observe that the PCE may not be suitable to describe the steady-state response of a random system harmonically excited at a frequency close to a deterministic eigenfrequency. Second, an explanation of this undesirable behavior is given. Third, the quantities of interest are studied when the number of terms of the PCE is increased; the behavior of the PCE around the deterministic resonances is highlighted. Finally, it is shown that a PCE may be possible even for a high PC order.

\section{Response of a Harmonically Excited Random Dynamical System}

\section{Uncertainties in a Dynamical System}

The dynamical system is assumed to be described by its $n \times n$ mass, damping, and stiffness matrices, $\mathbf{M}, \mathbf{D}$, and $\mathbf{K}$, where $n$ is the number of DOFs, the forces acting on this system are described by force vector $\mathbf{F}(t)$, and $\mathbf{x}(t)$ denotes the DOF vector.

The stiffness matrix is assumed to be uncertain and is given by

$$
\mathbf{K}=\overline{\mathbf{K}}\left(1+\delta_{K} \xi_{K}\right)
$$

where $\xi_{K}=$ standard normal deviate; and $\overline{\mathbf{K}}=$ deterministic matrix, which represents the mean stiffness matrix; the covariance matrix of $\mathbf{K}$ is controlled by parameter $\delta_{K}$. The deterministic dynamical system corresponds to $\delta_{K}=0$. This uncertainty is very simple. This choice was motivated by the objective of the paper, which is not to find a new method to derive the moments but to highlight and to explain a specific feature of a PCE around the deterministic eigenfrequencies. This class of uncertainty makes the equations and the explanations clearer. From a theoretical point of view, the normal distribution may not appear relevant as a stiffness must not be negative. However, because of the value of the mean and standard deviation (SD) of the proposed distribution $(\mathrm{SD}=0.05$ for a unit mean), a negative stiffness is an extremely rare event: for 10,000 independent samples of the random variable, no negative realization was achieved, and accordingly, the probability that the stiffness matrix defined by Eq. (1) is not positive definite is extremely weak. From a practical point of view, this distribution is relevant. Further, in a manufacturing context, the mean and SD are the only known statics; therefore, if the constraint of positiveness is disregarded, the distribution specified by the principle of maximum entropy is the normal distribution. It must also be specified that all the developments made in this paper are applicable to other distributions such as the uniform distribution.

In this study, the mass and damping matrices are assumed to be deterministic. However, this is not a restriction, and this study may be done with random mass and damping matrices. Conceptually, the natural frequencies could have been perturbed. In this way, the results of this study would immediately be generalized to proportionally damped systems.

The force vector is assumed to be harmonic $\mathbf{F}(t)=\mathbf{F}_{0} e^{I \omega t}$, and the steady-state response of the dynamical system is then $\mathbf{x}(t)=\mathbf{X} e^{I \omega t}$, where $I=\sqrt{-1}$, and $\mathbf{X}$ is the solution of the following equation:

$$
\left(-\omega^{2} \mathbf{M}+I \omega \mathbf{D}+\mathbf{K}\right) \mathbf{X}=\mathbf{F}_{0}
$$

As $\mathbf{K}=$ random matrix, $\mathbf{X}=$ random vector, which can be described by its moments. In this study, the first two moments are estimated. Because of the class of system under study, these moments may also be obtained by solving deterministic differential equations (Grigoriu 2002) or by conditional Monte Carlo (Grigoriu 2002; Hammersley 1956).

As discussed previously, several methods may be used to derive these moments, such as MCS or the PCE. In this paper, the MCS results will be the reference results and the PCE results will be compared to them.

The PC method is now a well-known and well-studied alternative to MCS that expands the solution $\mathbf{X}$ in terms of polynomials $\Psi_{j}$, whose variables are a set of mutually independent standard normal deviates (Ghanem and Spanos 1991). In this case, polynomial chaos $\Psi_{j}$ is a Hermite polynomial and depends on one random variable $\xi_{K}$. Therefore, the response can be expanded on the PC basis such that

$$
\mathbf{X}\left(\omega, \xi_{K}\right)=\sum_{i=0}^{\infty} \mathbf{X}_{i}(\omega) \Psi_{i}\left(\xi_{K}\right)
$$

This expression for $\mathbf{X}\left(\omega, \xi_{K}\right)$ can be truncated to a finite number of terms $P$ for the numerical study; $P$ defines the number of polynomials that is given by $P+1=(m+r) ! /(m ! r !)$, where $r$ is the number of random variables and $m$ is the chaos order. Therefore, by truncating the previous infinite expansion, an approximation of $\mathbf{X}\left(\omega, \xi_{K}\right)$ is obtained as

$$
\mathbf{X}^{P}\left(\omega, \xi_{K}\right)=\sum_{i=0}^{P} \mathbf{X}_{i}(\omega) \Psi_{i}\left(\xi_{K}\right)
$$

In the following, the argument $\xi_{K}$ of $\mathbf{X}^{P}$ is dropped for convenience.

\section{PC Component Dynamic Equation}

As already mentioned, DOF vector $\mathbf{x}(t)$ is a random vector, and $\mathbf{x}(t)$ is a solution to the equation

$$
\mathbf{M} \ddot{\mathbf{x}}(t)+\mathbf{D} \dot{\mathbf{x}}(t)+\mathbf{K x}(t)=\mathbf{F}(t)
$$

where $\mathbf{K}$ is defined by Eq. (1).

This random response vector may be expanded in terms of Hermite polynomials $\Psi_{j}$ (Ghanem and Spanos 1991; Dessombz 2000). An approximation is obtained by truncating the infinite series 


$$
\mathbf{x}^{P}(t)=\sum_{i=0}^{P} \mathbf{Y}_{i}(t) \Psi_{i}\left(\xi_{k}\right)
$$

$t$ will be dropped to shorten the equations.

Substituting Eq. (6) into Eq. (5), one has

$$
\sum_{i=0}^{P} \Psi_{j}\left(\xi_{K}\right)\left(\mathbf{M} \ddot{\mathbf{Y}}_{i}+\mathbf{D} \dot{\mathbf{Y}}_{i}+\mathbf{K} \mathbf{Y}_{i}\right)=\mathbf{F}
$$

By using the Hermite polynomial properties and by taking into account Eq. (1), the following equations are obtained:

$$
\begin{array}{r}
\forall j=0 \cdots P, \quad\langle 0, j, j\rangle\left(\mathbf{M} \ddot{\mathbf{Y}}_{j}+\mathbf{D} \dot{\mathbf{Y}}_{j}+\overline{\mathbf{K}} \mathbf{Y}_{j}\right) \\
+\sum_{i=0}^{P} \delta_{K}\langle 1, i, j\rangle \overline{\mathbf{K}} \mathbf{Y}_{\mathbf{j}}=\delta_{0 j} \mathbf{F}
\end{array}
$$

where $\delta_{i j}=$ Kronecker $\delta$ and

$$
\langle k, i, j\rangle=\int_{-\infty}^{+\infty} \xi_{K}^{k} \Psi_{i}\left(\xi_{K}\right) \Psi_{j}\left(\xi_{K}\right) \frac{e^{-\xi_{K}^{2} / 2}}{\sqrt{2 \pi}} \mathrm{d} \xi_{K}
$$

Because of the Hermite polynomial properties, the following relations may be easily derived:

$$
\begin{aligned}
\langle 0, i, j\rangle & =\int_{-\infty}^{+\infty} \Psi_{i}\left(\xi_{K}\right) \Psi_{j}\left(\xi_{K}\right) \frac{e^{-\xi_{K}^{2} / 2}}{\sqrt{2 \pi}} \mathrm{d} \xi_{K}=j ! \delta_{i j} \\
\langle 1, i, j\rangle & =\int_{-\infty}^{+\infty} \xi_{K} \Psi_{i}\left(\xi_{K}\right) \Psi_{j}\left(\xi_{K}\right) \frac{e^{-\xi_{K}^{2} / 2}}{\sqrt{2 \pi}} \mathrm{d} \xi_{K} \\
& =\delta_{j i+1} j !+\delta_{j i-1}(j+1) !
\end{aligned}
$$

Define

$$
\begin{gathered}
\mathbf{M o m}^{0} / \operatorname{Mom}_{i j}^{0}=\langle 0, i, j\rangle \text { and } \mathbf{M o m}^{0} \in \mathbb{R}^{(P+1) \times(P+1)} \\
\mathbf{M o m}^{1} / \operatorname{Mom}_{i j}^{1}=\langle 1, i, j\rangle \text { and } \mathbf{M o m}^{1} \in \mathbb{R}^{(P+1) \times(P+1)} \\
\tilde{\mathbf{M}}=\mathbf{M o m}^{0} \otimes \mathbf{M} \in \mathbb{R}^{2(P+1) \times 2(P+1)} \\
\tilde{\mathbf{D}}=\mathbf{M o m}^{0} \otimes \mathbf{D} \in \mathbb{R}^{2(P+1) \times 2(P+1)} \\
\tilde{\mathbf{K}}=\left(\mathbf{M o m}{ }^{0}+\delta_{K} \mathbf{M o m} \mathbf{m}^{1}\right) \otimes \overline{\mathbf{K}} \in \mathbb{R}^{2(P+1) \times 2(P+1)} \\
\mathbf{Y}=\left[\mathbf{Y}_{0}^{T} \mathbf{Y}_{1}^{T} \cdots \mathbf{Y}_{\mathbf{P}}^{T}\right]^{T} \in \mathbb{R}^{2(P+1)} \\
\tilde{\mathbf{F}}(t)=\left[\mathbf{F}^{T}(t) 00 \cdots 0\right]^{T} \in \mathbb{R}^{2(P+1)}
\end{gathered}
$$

where $\otimes=$ Kronecker product; and $(\bullet)^{T}=$ transpose of $(\bullet)$. Then, the components of the PC expansion satisfy the following equation:

$$
\tilde{\mathbf{M}} \ddot{\mathbf{Y}}(t)+\tilde{\mathbf{D}} \dot{\mathbf{Y}}(t)+\tilde{\mathbf{K}} \mathbf{Y}(t)=\tilde{\mathbf{F}}(t)
$$

It should be noted that Eq. (19) shows that the change of variables provided by Eq. (6) leads to a $2(P+1)$-DOF dynamical system that will be referred to as the PC system in the following. Therefore, the PCE transforms the study of a random dynamical system to the study of a deterministic dynamical system. Accordingly, the PC system has resonant frequencies and the steady-state response to a harmonic force shows peaks related to these spurious resonances, as will be illustrated with a 2-DOF system. This is an important result of this paper; the spurious resonances will be referred to as $\mathrm{PC}$ resonances.

\section{Example}

\section{2-DOF System}

The MCS and PCE methods will be used to evaluate the mean and SD of $\mathbf{X}$ for the example shown in Fig. 1 (Didier 2012). Stiffness $k$ is assumed to be uncertain

$$
k=\bar{k}\left(1+\delta_{K} \xi_{K}\right)
$$

Thus, the mean stiffness matrix is

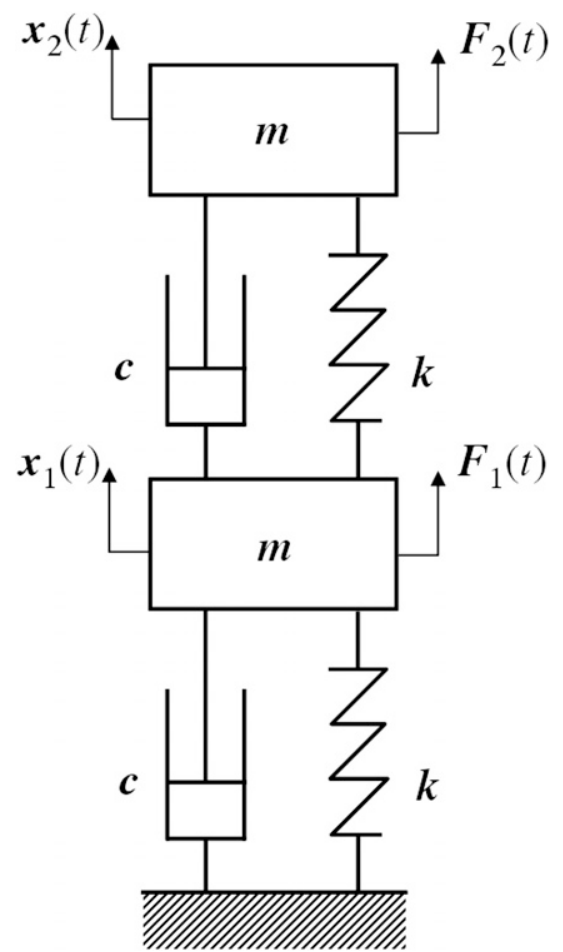

Fig. 1. 2-DOF system with stochastic stiffness coefficients

Table 1. System Characteristics

\begin{tabular}{lr}
\hline Characteristics & Value \\
\hline $\bar{k}\left(\mathrm{Nm}^{-1}\right)$ & 15,000 \\
$m(\mathrm{~kg})$ & 1 \\
$c\left(\mathrm{Nm}^{-1} \mathrm{~s}^{-1}\right)$ & 1 \\
$\delta_{K}($ percentage $)$ & 5 \\
$F_{01}(\mathrm{~N})$ & 1 \\
$F_{02}(\mathrm{~N})$ & 0 \\
\hline
\end{tabular}




$$
\overline{\mathbf{K}}=\bar{k}\left[\begin{array}{cc}
2 & -1 \\
-1 & 1
\end{array}\right]
$$

The characteristics of the system are listed in Tables 1 and 2 . The DOF vector was found for 501 values of $\omega /(2 \pi)$ in the range of $10-35 \mathrm{~Hz}(\Delta f=0.05 \mathrm{~Hz})$.

Table 2. Modal Characteristics of the Deterministic System

\begin{tabular}{lc}
\hline Eigenfrequencies, $f(\mathrm{~Hz})$ & Damping ratio, $\xi(\%)$ \\
\hline 12.05 & 0.25 \\
31.54 & 0.66 \\
\hline
\end{tabular}

The MCS results were obtained with 10,000 samples of the random variable $\xi_{K}$. The first two moments (mean and SD) are plotted in Fig. 2. The deterministic response is also plotted in Fig. 2.

The PC expansion is calculated for two PC orders: 2 and 15. The results are plotted in Figs. 3 and 4, and they are in perfect agreement with the MCS results, except around the resonances, even for $P=2$. Around the resonances, some spurious oscillations arise, and it seems that the curves oscillate more as $P$ increases.

As previously explained, the PC coefficients are the solution of a deterministic equation of motion of a mechanical system: the PC system. The DOF number of the PC system is a multiple of the DOF number of the deterministic real system; therefore, the eigenfrequencies of the PC system are noticeable in the steadystate response and resemble spurious PC eigenfrequencies. By solving the PC eigenproblem associated with matrices $(\tilde{\mathbf{K}}, \tilde{\mathbf{M}})$,

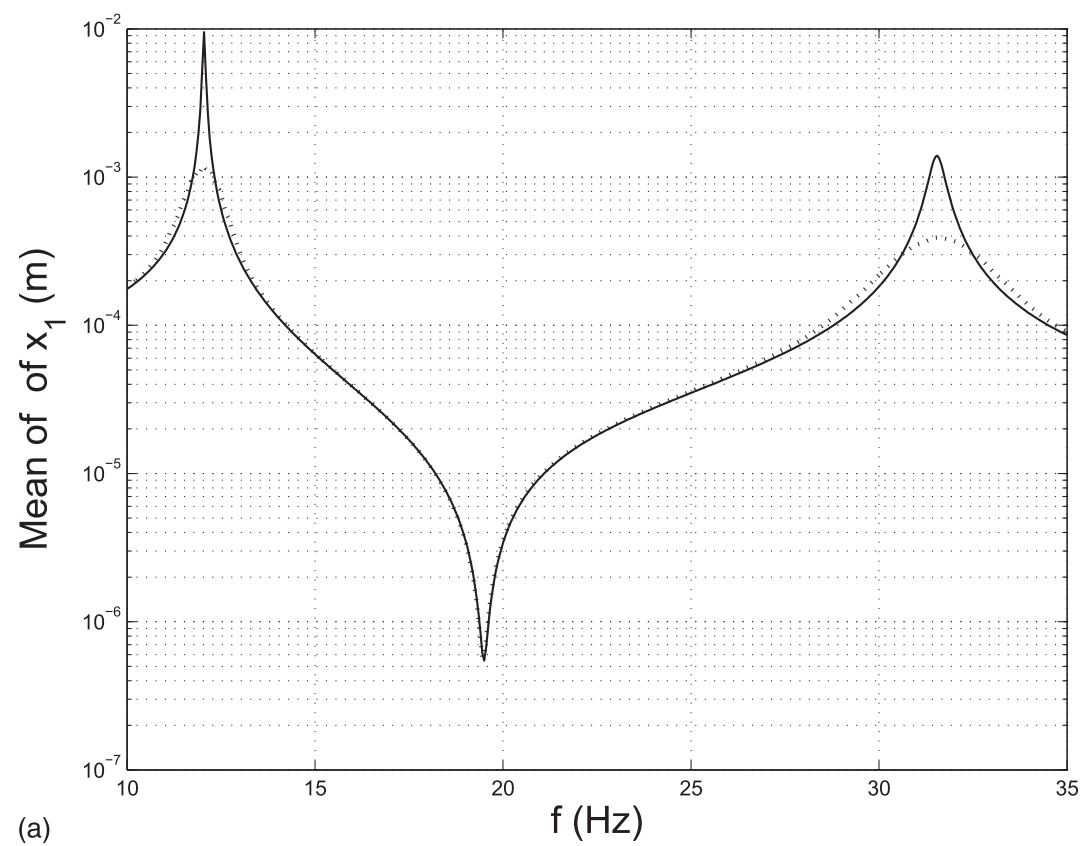

(a)

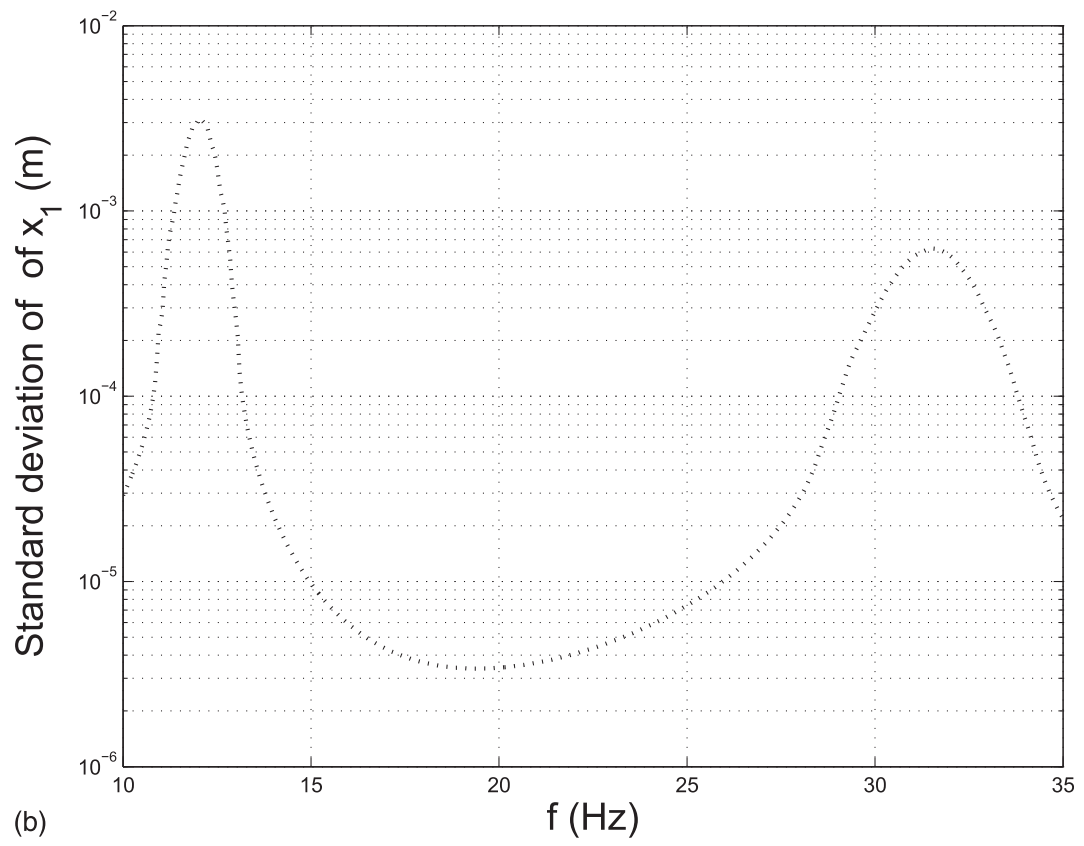

Fig. 2. Deterministic responses (solid lines); MCS with 10,000 samples (dotted lines): (a) mean of $x_{1}$; (b) SD of $x_{1}$ 


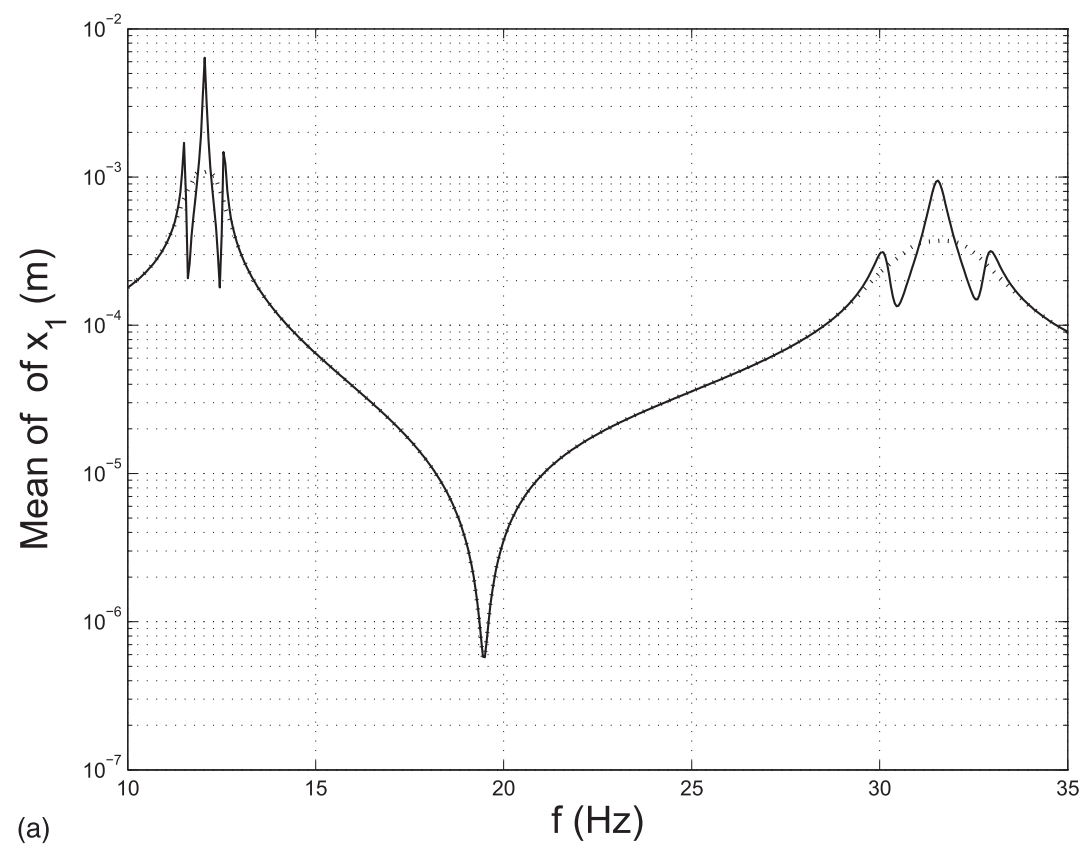

(a)

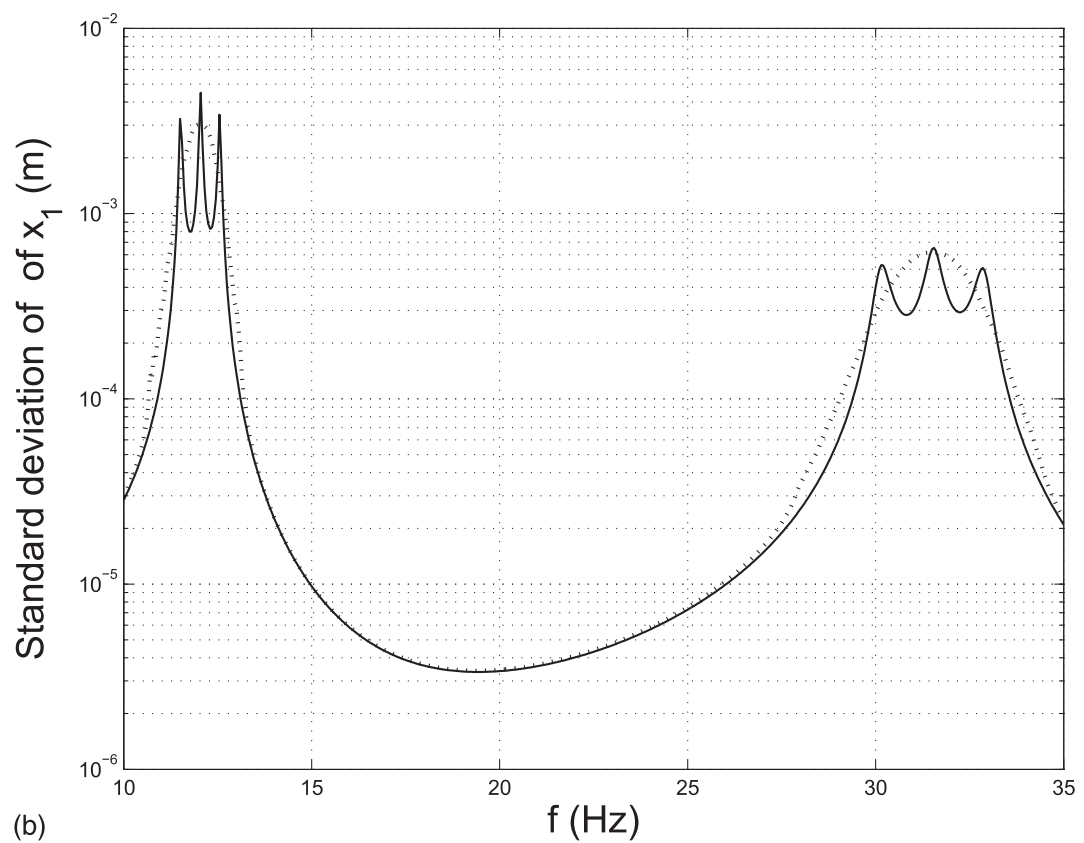

Fig. 3. PC expansion of order 2 (solid lines) versus MCS (dotted lines): (a) mean of $x_{1}$; (b) SD of $x_{1}$

the eigenfrequencies were determined, and then the modal damping ratios were calculated; the eigenfrequencies and modal damping ratios for several PC expansion degrees are listed in Table 3.

Table 3 shows that the PC resonant frequencies may be divided into two sets that are centered around the eigenfrequencies of the deterministic system. This explains why there seems to be more and more oscillations around the deterministic frequencies when $P$ increases (Figs. 3 and 4). In fact, there are no oscillations, but the peaks are associated with resonances that have no physical meaning, and the number of resonances increases with $P$. Similarly, the damping ratios associated with a set of eigenfrequencies are similar to the corresponding damping ratio. This explains why the curve is smoother around the second deterministic eigenfrequency than the first one.
Moreover, it turns out that the deterministic frequencies are in the set of eigenfrequencies only when $P$ is even. When $P$ is odd, the deterministic frequencies are antiresonant frequencies. This fact is illustrated in Fig. 5 around the first and second deterministic frequencies of the system. Not only are fluctuations of the mean amplitudes observed when $P$ increases, but also there are decreases in the amplitudes and a switch between high and low amplitudes at the first and second deterministic frequencies. This will be explained in the following.

\section{1-DOF System}

The study of a random 1-DOF system is now required to go further in the calculation, and it shows representative behavior and the underlying physics that would not change significantly with higher 


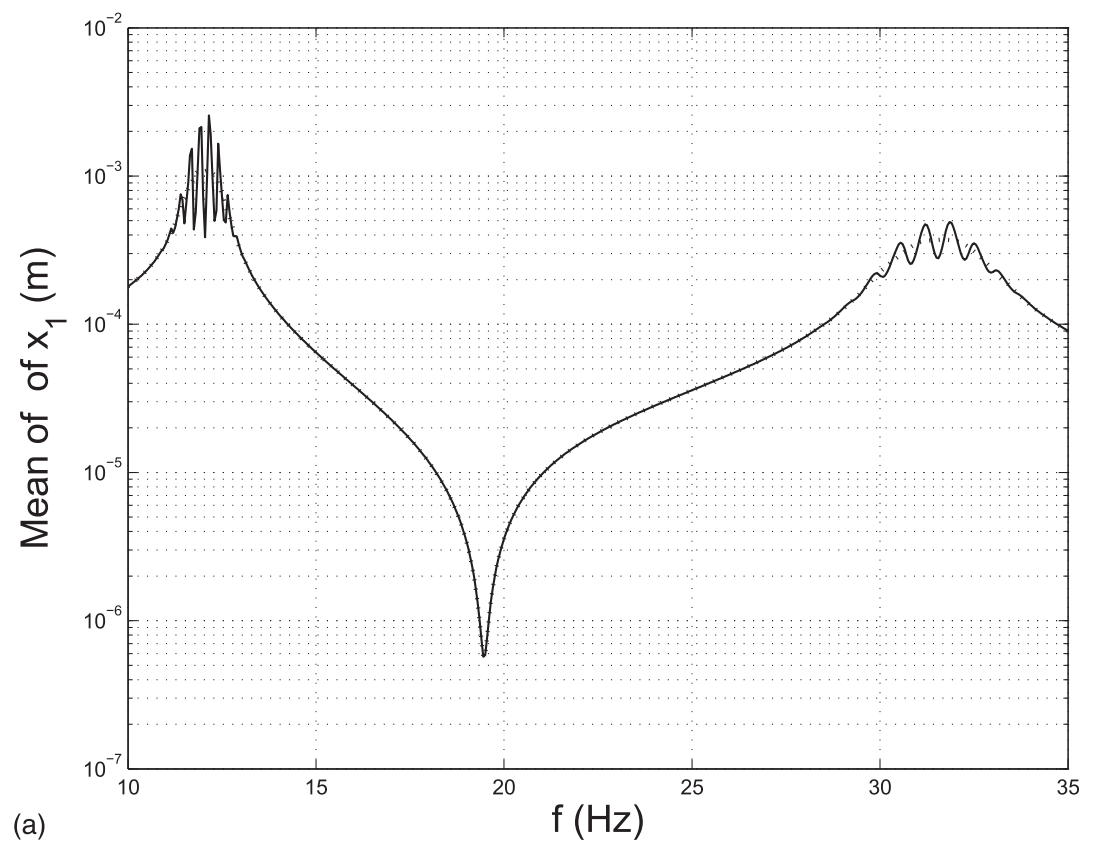

(a)

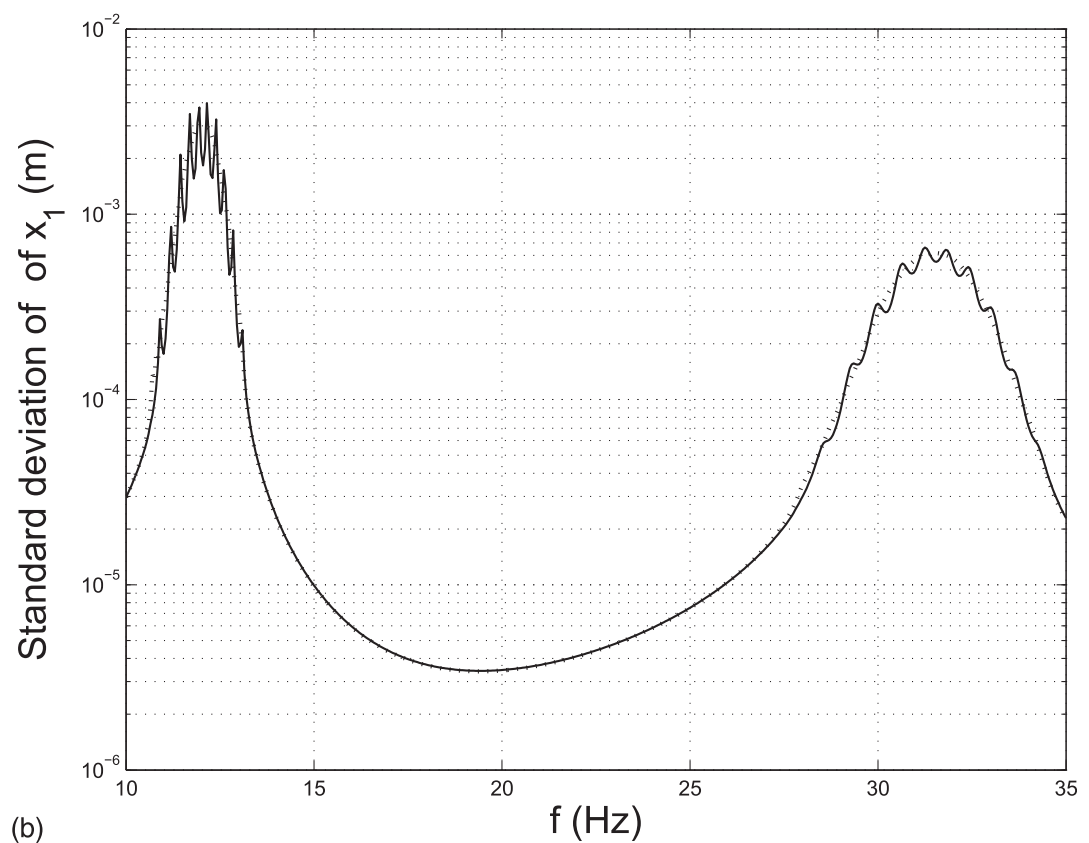

Fig. 4. PC expansion of order 15 (solid lines) versus MCS (dotted lines): (a) mean of $x_{1}$; (b) SD of $x_{1}$

dimension. Therefore, to keep working on the same example, a modal analysis is performed and the modal coordinates are developed on PCs.

Let's note $\left(\omega_{\text {det }}^{(i)}, \boldsymbol{\Phi}_{\text {det }}^{(i)}\right)$ the deterministic $i$ th mode associated with $(\overline{\mathbf{K}}, \mathbf{M}): \boldsymbol{\Phi}_{\operatorname{det}}^{(i)}$ is also an eigenvector of $(\mathbf{K}, \mathbf{M})$ because of Eq. (20).

In the following, modal quantity $(\bullet)$ related to mode $i$ will be denoted $(\bullet)^{(i)}$. The associated modal coordinate, $q^{(i)}(t)$, satisfies

$$
m^{(i)} \ddot{q}^{(i)}+c^{(i)} \dot{q}^{(i)}+k^{(i)} q^{(i)}=F^{(i)}
$$

where $F^{(i)}=\Phi_{\operatorname{det}}^{(i) T} \mathbf{F}$; and $m^{(i)}, c^{(i)}$, and $k^{(i)}$ may be easily determined from the system characteristics. Moreover, Eq. (20) means that $k^{(i)}=\bar{k}^{(i)}\left(1+\delta_{K} \xi_{K}\right)$.
Suppose a harmonic force $\left[\mathbf{F}_{0} \exp (I \omega t)\right]$ acts on the mass, and the steady-state response is $q^{(i)}(t)=Q^{(i)} \exp (I \omega t) ; Q^{(i)}$ is expanded on the PCs

$$
Q^{(i)}=\sum_{j=0}^{P} Y_{j}^{(i)} \Psi_{j}\left(\xi_{K}\right)
$$

and $Q^{(i)}$ is governed by the following equation:

$$
\left(a^{(i)}+\bar{k}^{(i)} \delta_{K} \xi_{K}\right) Q^{(i)}=F^{(i)}
$$

where $a^{(i)}=\left(\bar{k}^{(i)}-\omega^{2} m^{(i)}+I \omega c^{(i)}\right)$. Then, the PC expansion components satisfy 
Table 3. Modal Characteristics of the PC Component System

\begin{tabular}{|c|c|c|}
\hline PC order & $f(\mathrm{~Hz})$ & $\xi(\%)$ \\
\hline \multirow[t]{4}{*}{$P=1$} & 11.74 & 0.26 \\
\hline & 12.34 & 0.25 \\
\hline & 30.74 & 0.67 \\
\hline & 32.32 & 0.64 \\
\hline \multirow[t]{6}{*}{$P=2$} & 11.51 & 0.26 \\
\hline & 12.05 & 0.25 \\
\hline & 12.56 & 0.24 \\
\hline & 30.14 & 0.69 \\
\hline & 31.54 & 0.66 \\
\hline & 32.88 & 0.63 \\
\hline \multirow[t]{8}{*}{$P=3$} & 11.32 & 0.27 \\
\hline & 11.82 & 0.26 \\
\hline & 12.27 & 0.25 \\
\hline & 12.73 & 0.24 \\
\hline & 29.64 & 0.7 \\
\hline & 30.95 & 0.67 \\
\hline & 32.12 & 0.65 \\
\hline & 33.33 & 0.63 \\
\hline \multirow[t]{10}{*}{$P=4$} & 11.15 & 0.27 \\
\hline & 11.63 & 0.26 \\
\hline & 12.05 & 0.25 \\
\hline & 12.45 & 0.24 \\
\hline & 12.88 & 0.24 \\
\hline & 29.2 & 0.71 \\
\hline & 30.45 & 0.68 \\
\hline & 31.54 & 0.66 \\
\hline & 32.59 & 0.64 \\
\hline & 33.72 & 0.62 \\
\hline
\end{tabular}

$$
a^{(i)} \sum_{j=0}^{P} Y_{j}^{(i)} \Psi_{j}\left(\xi_{K}\right)+\bar{k}^{(i)} \delta_{K} \sum_{j=0}^{P} Y_{j}^{(i)} \xi_{K} \Psi_{j}\left(\xi_{K}\right)=F^{(i)}
$$

Using the Hermite polynomial orthogonality properties given by Eqs. (10) and (11), the following $P+1$ equations are derived:

$$
\begin{gathered}
a^{(i)} 0 ! Y_{0}^{(i)}+\bar{k}^{(i)} \delta_{K} 1 ! Y_{1}^{(i)}=F^{(i)} \\
a^{(i)} 1 ! Y_{1}^{(i)}+\bar{k}^{(i)} \delta_{K} 1 ! Y_{0}^{(i)}+\bar{k}^{(i)} \delta_{K} 2 ! Y_{2}^{(i)}=0 \\
\vdots=\vdots \\
a^{(i)}(P-1) ! Y_{P-1}^{(i)}+\bar{k}^{(i)} \delta_{K}(P-1) ! Y_{P-2}^{(i)}+\bar{k}^{(i)} \delta_{K} P ! Y_{P}^{(i)}=0 \\
a^{(i)} P ! Y_{P}^{(i)}+\bar{k}^{(i)} \delta_{K} P ! Y_{P-1}^{(i)}=0
\end{gathered}
$$

The $j$ th equation may be simplified by dividing this equation by $(j-1)$ !.

Define $N_{j}^{(i)}$ and $D_{j}^{(i)}$ as

$$
Y_{j}^{(i)}=\frac{N_{j}^{(i)}}{D_{j}^{(i)}} Y_{j-1}^{(i)}
$$

Then, the following recurrence relations hold:

$$
\begin{aligned}
& \forall j=2 \cdots P, \quad N_{j-1}^{(i)}=-\delta_{K} D_{j}^{(i)} \\
& \quad \text { and } \quad D_{j-1}^{(i)}=\frac{a^{(i)}}{\bar{k}^{(i)}} D_{j}^{(i)}+\delta_{K} j N_{j}^{(i)}
\end{aligned}
$$

with the initial values

$$
N_{P}^{(i)}=-\delta_{K} \quad \text { and } \quad D_{P}^{(i)}=\frac{a^{(i)}}{\bar{k}^{(i)}}
$$

Then, $N_{1}^{(i)}$ and $D_{1}^{(i)}$ may be derived easily, and finally

$$
Y_{0}^{(i)}=\frac{D_{1}^{(i)}}{a^{(i)} D_{1}^{(i)}+\bar{k}^{(i)} \delta_{K} N_{1}^{(i)}} F^{(i)}
$$

By mathematical induction, it is easy to prove that $a^{(i)}$ may be factorized either in $N_{j}^{(i)}$ or in $D_{j}^{(i)}$ for each $j$. More precisely, it may be shown that if $a^{(i)}$ is a factor of $D_{j}^{(i)}$ (respectively, $N_{j}^{(i)}$ ), then $a^{(i)}$ is a factor of $N_{j-1}^{(i)}$ (respectively, $D_{j-1}^{(i)}$ ). However, $\omega_{\mathrm{det}}^{(i)}$ is a resonant frequency of the frequency response function (FRF) $1 / a^{(i)}$; accordingly, $\omega_{\text {det }}^{(i)}$ is a resonant frequency of $Y_{P}^{(i)}, Y_{P-2}^{(i)}, Y_{P-4}^{(i)}$, and so on. Similarly, $\omega_{\text {det }}^{(i)}$ is an antiresonant frequency of $Y_{P-1}^{(i)}, Y_{P-3}^{(i)}, Y_{P-5}^{(i)}$, and so on. Then if $P$ is even, $\omega_{\mathrm{det}}^{(i)}$ is a resonant frequency of $Y_{0}^{(i)}$, which corresponds to the mean of the $i$ th modal response; if $P$ is odd, $\omega_{\text {det }}^{(i)}$ is an antiresonant frequency of $Y_{0}^{(i)}$. The change of variable $\mathbf{X}^{P}=\boldsymbol{\Phi}_{\mathrm{det}}^{T} \mathbf{Q}$ leads to the same conclusions on the steady-state response.

This also shows that the behavior of the response expanded on the PC depends on the parity of $P$. However, PCE converges in probability and distribution (Ghanem and Spanos 1991; Field and Grigoriu 2007), so $\mathbf{X}^{2 P}\left(\omega=\omega_{\mathrm{det}}\right)$ and $\mathbf{X}^{2 P+1}\left(\omega=\omega_{\mathrm{det}}\right)$ tend to the same limit as $P$ tends to infinity. Define the following discrepancy for the first DOF:

$$
\mathcal{D}(P)=\frac{\left\|X_{1 \text { mean }}^{P+1}-X_{1 \text { mean }}^{P}\right\|}{\left\|X_{1 \text { mean }}^{P}\right\|}
$$

This discrepancy provides valuable information about the required number of terms to include in the expansion. Fig. 6(a) shows that for low $P$, this discrepancy varies significantly from $P$ to $P+1$ because of the alternating resonances and antiresonances. However, for $P>300, \mathcal{D}(P)$ is approximately $10 \%$. Fig. 7(a) shows that for $P=100$, the peaks are still noticeable around the first eigenfrequency, whereas the agreement with the MCS results are excellent for $P=300$ [cf. Fig. 7(b)]. It is also interesting to note the PC damping effect; as the $\mathrm{PC}$ damping ratio associated with the second deterministic mode is higher than for the first mode, the agreement between PCE and MCS around the second mode is good for a PC order lower than 300 . Indeed, no oscillations are noticeable around the second mode in Fig. 7(a), that is, for $P=100$.

However, in practice, a convergence is not purely theoretical; it must be possible to be close enough to the limit from a numerical point of view. This may be a real problem for a very low damping ratio. In that case, when the system is excited at a frequency equal to a deterministic eigenfrequency, the alternating resonance and antiresonance will produce a sequence with alternatively very low and very high values. Then, a very high $P$ will be required. In Fig. 6(b), discrepancy $\mathcal{D}$ was plotted when the damping is divided by 5 , that is, for a first mode damping ratio equal to $0.05 \%$. Even for $P$ equal to 1,500 , the limit is not reached. Unfortunately, for $P$ higher than 1,800 , some numerical problems occurred, and it was not possible to have a good estimation of the limit.

The use of the recurrence relation [Eq. (31)] also provides an interesting expression for $Y_{j}^{(i)}$ 


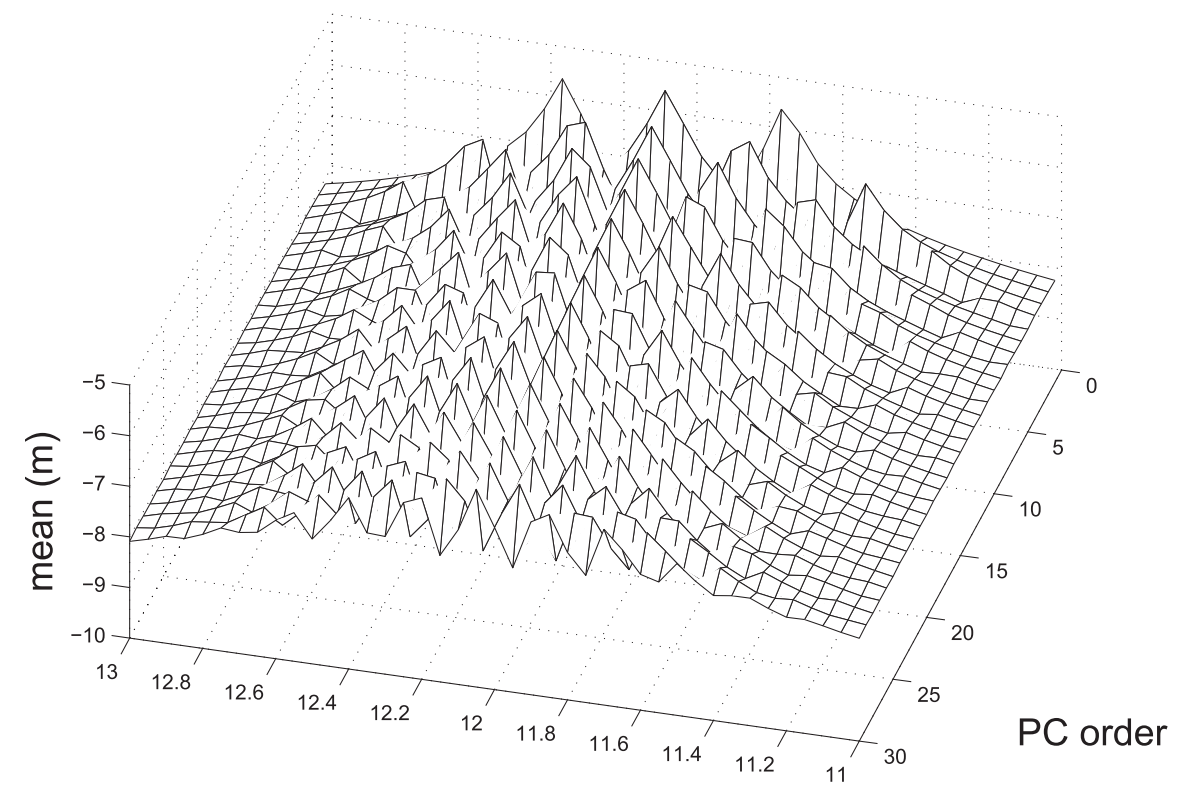

(a)

frequency $(\mathrm{Hz})$

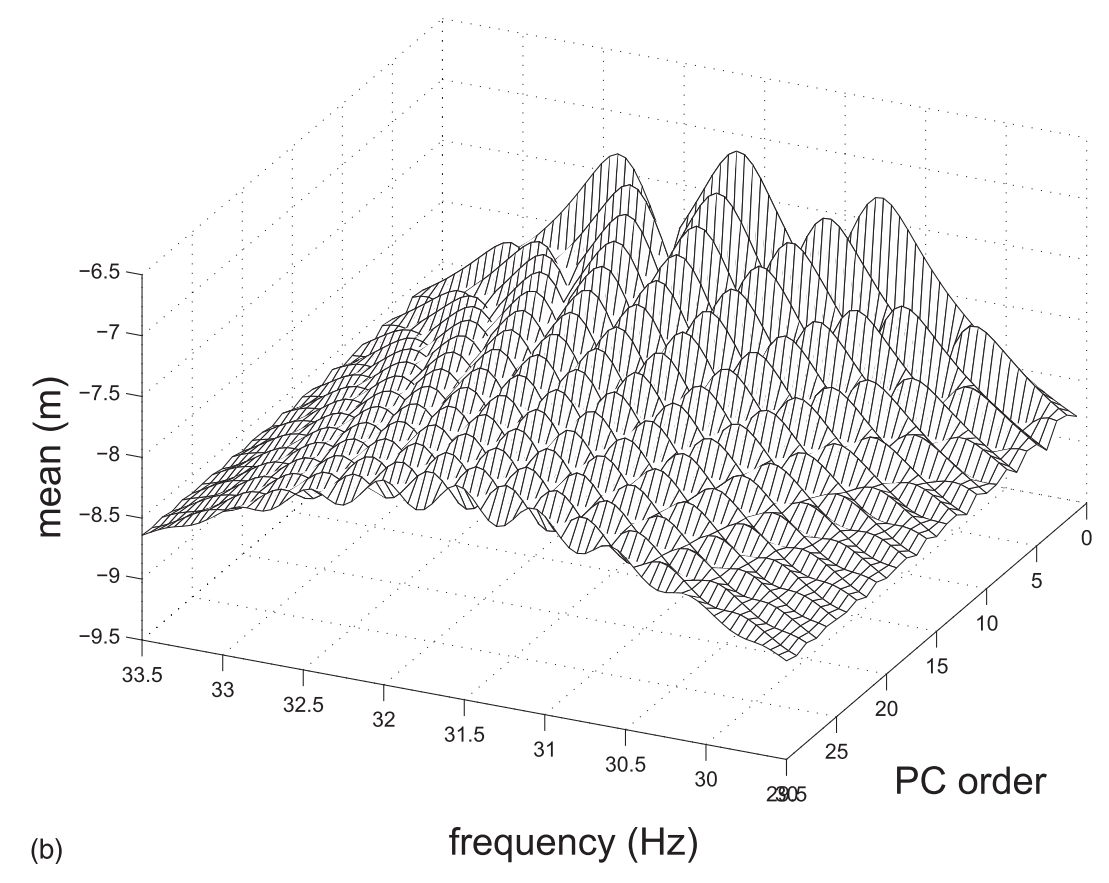

Fig. 5. Behavior around the (a) first and (b) second deterministic eigenfrequencies for several PC orders

$$
\begin{aligned}
\forall j \geq 1, \quad Y_{j}^{(i)} & =\frac{N_{j}^{(i)}}{D_{j}^{(i)}} Y_{j-1}^{(i)}=\frac{N_{j}^{(i)}}{D_{j}^{(i)}} \frac{N_{j-1}^{(i)}}{D_{j-1}^{(i)}} \cdots \frac{N_{1}^{(i)}}{D_{1}^{(i)}} Y_{0}^{(i)} \\
& =\frac{N_{j}^{(i)}}{D_{j}^{(i)}} \frac{N_{j-1}^{(i)}}{D_{j-1}^{(i)}} \cdots \frac{N_{1}^{(i)}}{D_{1}^{(i)}} \frac{D_{1}^{(i)}}{a^{(i)} D_{1}^{(i)}+\delta_{K} \bar{k}_{i}^{\mathrm{mod}} N_{1}^{(i)}} F_{i}^{\mathrm{mod}} \\
& =\frac{N_{j}^{(i)}}{D_{j}^{(i)}} \frac{-\delta_{K} D_{j}^{(i)}}{D_{j-1}^{(i)}} \cdots \frac{-\delta_{K} D_{2}^{(i)}}{D_{1}^{(i)}} \frac{D_{1}^{(i)}}{a^{(i)} D_{1}^{(i)}+\delta_{K} \bar{k}_{i}^{\bmod } N_{1}^{(i)}} F_{i}^{\mathrm{mod}} \\
& =\left(-\delta_{K}\right)^{j-1} \frac{N_{j}^{(i)}}{a^{(i)} D_{1}^{(i)}+\delta_{K} \bar{k}_{i}^{\bmod } N_{1}^{(i)}} F_{i}^{\bmod }
\end{aligned}
$$

Then the modal coordinates may be obtained as

$$
Q^{(i)}=\frac{F_{i}^{\mathrm{mod}}}{a^{(i)} D_{1}^{(i)}+\delta_{K} \bar{k}_{i}^{\mathrm{mod}} N_{1}^{(i)}}\left[D_{1}^{(i)} \Psi_{0}+\sum_{j=1}^{P}\left(-\delta_{K}\right)^{j-1} N_{j}^{(i)} \Psi_{j}\right]
$$

\section{Conclusion}

The comparison between MCS and a PCE of a random system steadystate response statistics showed that the PCE results converge quickly to the MCS results except around the deterministic eigenfrequencies. This study proved that these oscillations are caused by 

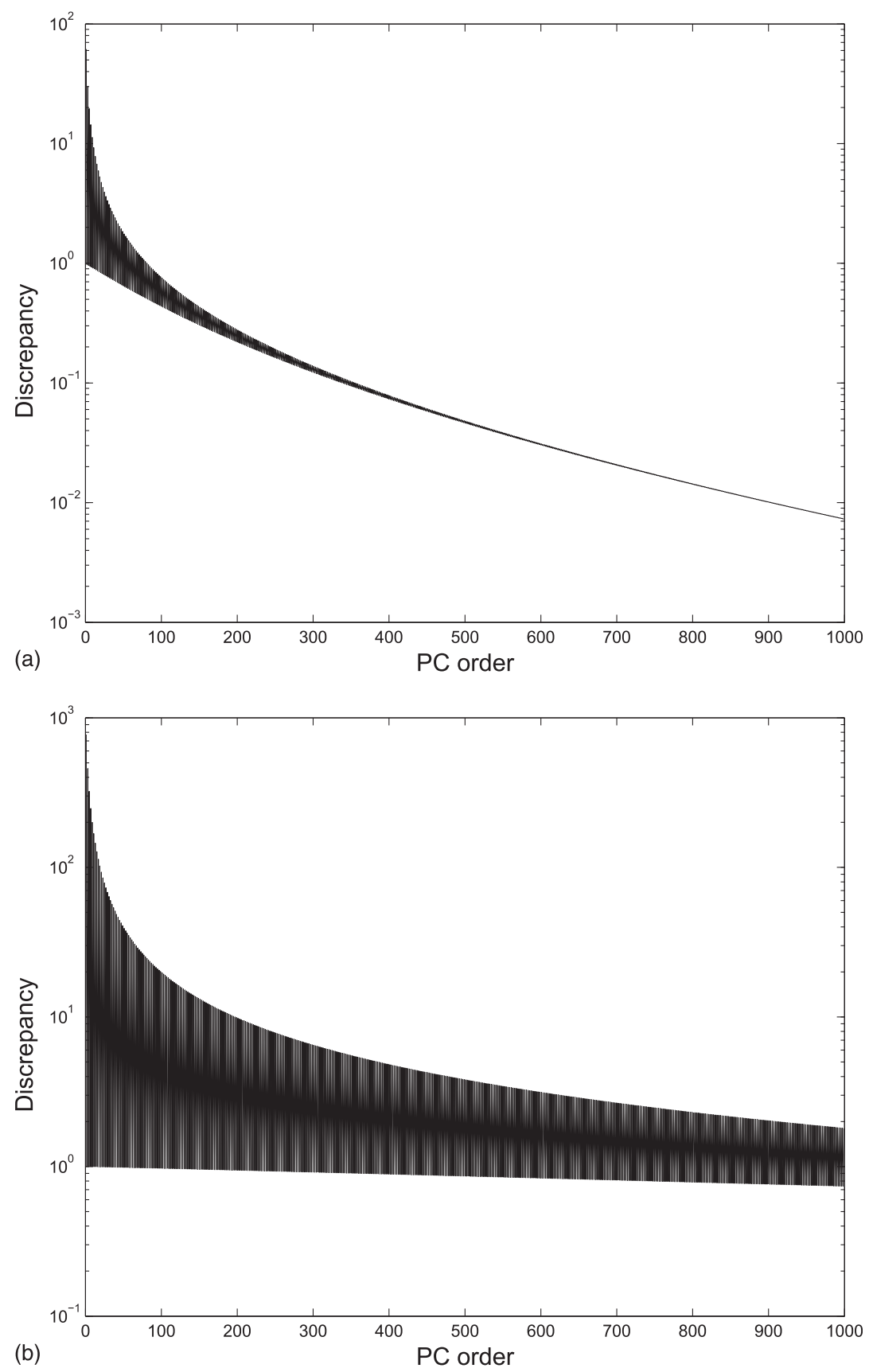

Fig. 6. Discrepancy for increasing PC order: (a) damping ratio $\xi=0.25 \%$; (b) damping ratio $\xi=0.05 \%$

peaks that correspond to resonances. Indeed, the PCE coefficients are the response of a deterministic dynamical system that has $P+1$ eigenfrequencies located around each deterministic eigenfrequency of the actual system; this explains why the spurious oscillations increase with the PC order. This result shows that the PCE coefficients are calculated by transforming the initial deterministic dynamical system into another dynamical system.

Moreover, it was observed that the PCE does not tend monotonically to the MCS around the deterministic frequencies; this depends on the parity of the PC order. More precisely, when the random system is excited at a deterministic frequency, the response moments are associated either with a resonance for an even PC order or with an antiresonance for an odd PC order. Accordingly, it may be very slow to reach the MCS results for low damping ratios, and this may not be numerically achieved. A discrepancy was defined and provides information about the number of PCs that must be used.

The response of a harmonically excited random system can take advantage of modal analysis to determine a PCE. Indeed, the equations may be simplified, and some recurrence relations were determined. It was then possible to achieve a PCE even for a high PC order that provided results very quickly, which are in an excellent agreement with the MCS results.

The results are related to a class of uncertain dynamical systems. This may be easily extended to uncertain mass, damping, and stiffness matrices that have a Karhunen-Loève expansion, because of the orthogonality properties of the multidimensional PC. In this study, the number of DOFs was small. The analysis of more realistic systems is the subject of ongoing research. Moreover, an effort must 


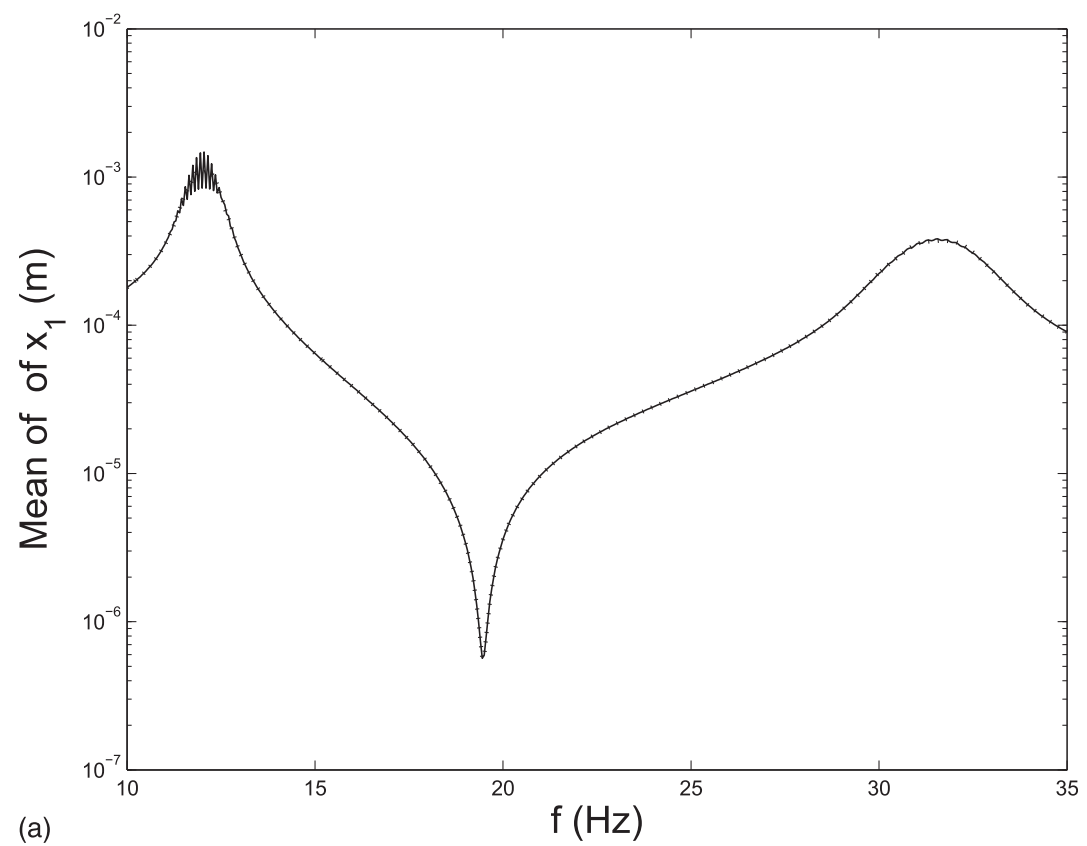

(a)

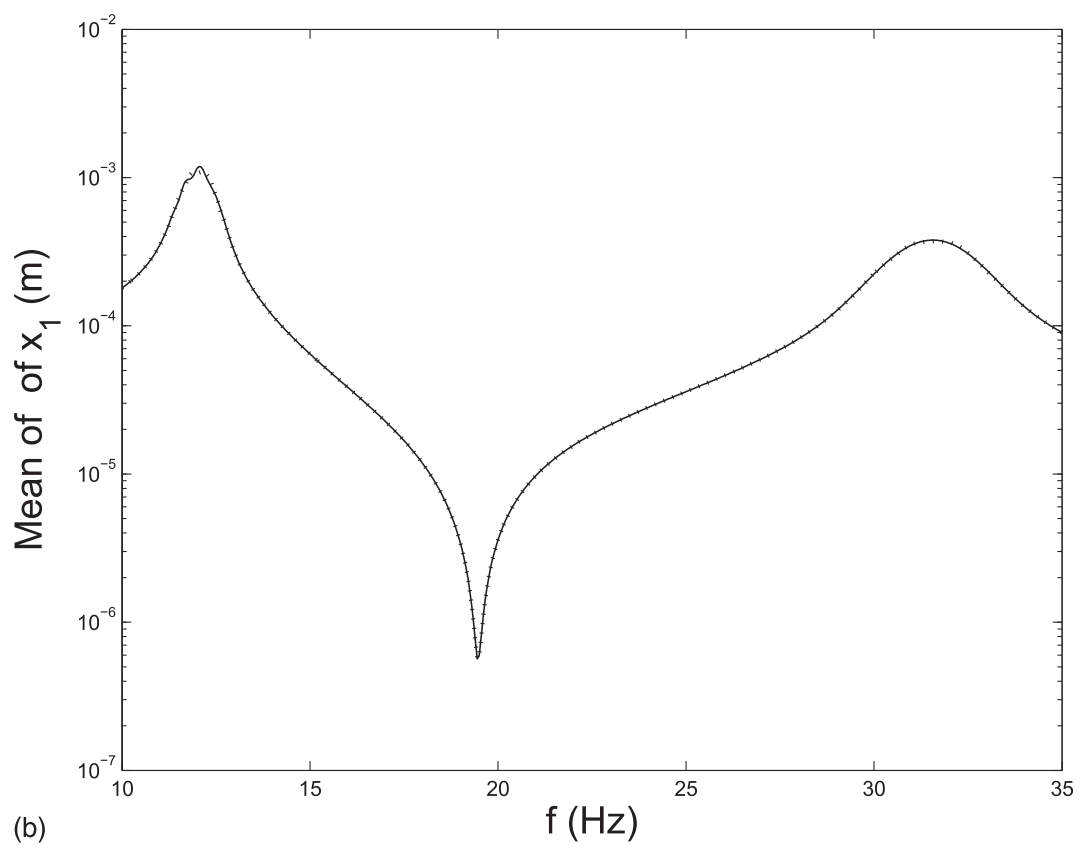

Fig. 7. Mean of $x_{1}$ for (a) $P=100$ and (b) $P=300$ : PC expansion (solid lines) versus MCS (dotted lines)

be made to calculate the PCE efficient at each excitation frequency; work is ongoing to deal with that issue.

\section{Acknowledgments}

S. Adhikari acknowledges the support of the Royal Society of London through the Wolfson Research Merit award.

\section{References}

Adhikari, S. (2011). "Doubly spectral stochastic finite-element method for linear structural dynamics." J. Aerosp. Eng., 10.1061/(ASCE)AS.19435525.0000070, 264-276.
Adhikari, S., and Manohar, C. S. (1999). "Dynamic analysis of framed structures with statistical uncertainties." Int. J. Numer. Methods Eng., 44(8), 1157-1178.

Adhikari, S., and Manohar, C. S. (2000). "Transient dynamics of stochastically parametered beams." J. Eng. Mech., 10.1061/(ASCE)07339399(2000)126:11(1131), 1131-1140.

Berveiller, M., Sudret, B., and Lemaire, M. (2006). "Stochastic finite elements: A non intrusive approach by regression." Eur. J. Comput. Mech., 15(1-3), 81-92.

Dessombz, O. (2000). "Analyse dynamique de structures comportant des paramètres incertains.” Ph.D. thesis, École Centrale de Lyon, Lyon, France.

Didier, J. (2012). "Dynamique des structures non-linéaires en présence d'incertitudes." Ph.D. thesis, École Centrale de Lyon, Lyon, France.

Falsone, G., and Ferro, G. (2005). "A method for the dynamical analysis of FE discretized uncertain structures in the frequency domain." Comput. Methods Appl. Mech. Eng., 194(42-44), 4544-4564. 
Falsone, G., and Ferro, G. (2007). "An exact solution for the static and dynamic analysis of FE discretized uncertain structures." Comput. Methods Appl. Mech. Eng., 196(21-24), 2390-2400.

Fang, Z. (1995). "Dynamic analysis of structures with uncertain parameters using the transfer matrix method." Comput. Struct., 55(6), 1037 1044.

Field, R. V., Jr., and Grigoriu, M. (2004). "On the accuracy of the polynomial chaos approximation." Probab. Eng. Mech., 19(1-2), 65-80.

Field, R. V., Jr., and Grigoriu, M. D. (2007). "Convergence properties of polynomial chaos approximations for $L_{2}$ random variables." SAND20071262, Sandia National Laboratories, Albuquerque, NM.

Ghanem, R., and Spanos, P. (1991). Stochastic finite elements: A spectral approach, Springer-Verlag, New York.

Ghosh, D., Ghanem, R. G., and Red-Horse, J. (2005). "Analysis of eigenvalues and modal interaction of stochastic systems." AIAA J., 43(10), 2196-2201.

Grigoriu, M. (2002). Stochastic calculus: Applications in science and engineering, Birkhauser, Boston.

Hammersley, J. M. (1956). "Conditional Monte Carlo." J. Assoc. Comput. Mach., 3(2), 73-76.

Hurtado, J. E., and Barbat, A. H. (1998). "Monte Carlo techniques in computational stochastic mechanics." Arch. Comput. Methods Eng., 5(1), 3-29.

Impollonia, N., and Ricciardi, G. (2006). "Explicit solutions in the stochastic dynamics of structural systems." Probab. Eng. Mech., 21(2), 171-181.

Kleiber, M., and Hien, T. D. (1992). The stochastic finite element method, Wiley, Chichester, U.K.

Kundu, A., and Adhikari, S. (2014). "Transient response of structural dynamic systems with parametric uncertainty." J. Eng. Mech., 10.1061/ (ASCE)EM.1943-7889.0000643, 315-331.

Lucor, D., and Karniadakis, G. E. (2004). "Adaptive generalized polynomial chaos for nonlinear random oscillators." J. Sci. Comput., 26(2), $720-735$.

Manohar, C. S., and Adhikari, S. (1998). "Statistics of vibration energy flow in randomly parametered trusses." J. Sound Vib., 217(1), 43-74.

Muscolino, G., Ricciardi, G., and Impollonia, N. (2000). "Improved dynamic analysis of structures with mechanical uncertainties under deterministic input." Probab. Eng. Mech., 15(2), 199-212.
Nouy, A. (2009). "Recent developments in spectral stochastic methods for the numerical solution of stochastic partial differential equations." Arch. Comput. Methods Eng., 16(3), 251-285.

Ostoja-Starzewski, M., and Woods, A. (2003). "Spectral finite elements for vibrating rods and beams with random field properties." J. Sound Vib., 268(4), 779-797.

Papadimitriou, C., Beck, J. L., and Katafygiotis, L. S. (1997). “Asymptotic expansions for reliability and moments of uncertain systems." J. Eng. Mech., 10.1061/(ASCE)0733-9399(1997)123:12(1219), 1219-1229.

Pascual, B., and Adhikari, S. (2012). "Hybrid perturbation-Polynomial Chaos approaches to the random algebraic eigenvalue problem." Comput. Methods Appl. Mech. Eng., 217-220(1), 153-167.

Pichler, L., Pradlwarter, H. J., and Schuëller, G. I. (2009). "A mode-based meta-model for the frequency response functions of uncertain structural systems." Comput. Struct., 87(5-6), 332-341.

Pradlwarter, H. J., and Schuëller, G. I. (2010). "Uncertain linear structural systems in dynamics: Efficient stochastic reliability assessment." Comput. Struct., 88(1-2), 74-86.

Schuëller, G. I., and Pradlwarter, H. J. (2009). "Uncertain linear systems in dynamics: Retrospective and recent developments by stochastic approaches." Eng. Struct., 31(11), 2507-2517.

Stefanou, G. (2009). "The stochastic finite element method: Past, present and future." Comput. Methods Appl. Mech. Eng., 198(9-12), 1031-1051.

Udwadia, F. E. (1987a). "Response of uncertain dynamic systems. I." Appl. Math. Comput., 22(2-3), 115-150.

Udwadia, F. E. (1987b). "Response of uncertain dynamic systems. II.” Appl. Math. Comput., 22(2-3), 151-187.

Van den Nieuwenhof, B., and Coyette, J.-P. (2003). "Modal approaches for the stochastic finite element analysis of structures with material and geometric uncertainties." Comput. Methods Appl. Mech. Eng., 192(33-34), 3705-3729.

Verhoosel, C. V., Gutiérrez, M. A., and Hulshoff, S. J. (2006). "Iterative solution of the random eigenvalue problem with application to spectral stochastic finite element systems." Int. J. Numer. Methods Eng., 68(4), 401-424.

Xiu, D. (2007). "Efficient collocational approach for parametric uncertainty analysis." Commun. Comput. Phys., 2(2), 293-309.

Xiu, D., and Hesthaven, J. (2005). "High-order collocation methods for differential equations with random inputs." J. Sci. Comput., 27(3), 1118-1139. 